\title{
Learning Curve from 100 Cases of Totally Thoracoscopic Mitral Valve Replacement
}

\author{
Guan-hua Fang, $\mathbf{M D}^{1,2}$, Jin-hua Chen, $\mathrm{MD}^{1,2}$, Xiao-fu Dai, $\mathrm{MD}^{1,2}$ \\ ${ }^{1}$ Department of Cardiovascular Surgery, , Union Hospital, Fujian Medical University, Fujian Medical University, Fuzhou, China; \\ ${ }^{2}$ Key Laboratory of Cardio-Thoracic Surgery, Fujian Province University, Fuzhou, China
}

\section{ABSTRACT}

Background: To investigate and analyze the learning curve of totally thoracoscopic mitral valve replacement and provide a quantitative reference for cardiac surgeons to carry out the operation step by step.

Methods: The clinical data were retrospectively analyzed of 100 consecutive patients with totally thoracoscopic mitral valve replacement successively performed by the same surgeon in a single center from May 2019 to June 2020. The learning curve was divided into 2 stages by using cumulative sum analysis, and relevant surgical parameters and perioperative indicators were analyzed.

Results: The first stage of the learning curve is the skill acquisition stage, which includes 1 to 40 surgical procedures. The second stage is the proficiency stage, involving 41 to 100 operations. Among the surgical parameters of the patients in the 2 stages, detectable improvements were observed in operative time, cardiopulmonary bypass time, cross-clamp time, and intraoperative injury. After surgery, the amount of drainage, length of hospital stay, blood creatinine levels, and oxygenation index $24 \mathrm{~h}$ after surgery were also significantly different between the 2 groups (all $P<.05$ ). The age and sex distributions of the patients were balanced, and there was no statistically significant difference in terms of conversion to median sternotomy between the 2 stages $(P>.05)$.

Conclusions: Cumulative sum analysis was used to accurately analyze the learning curve of totally thoracoscopic mitral valve replacement, indicating that 40 cases are needed to master the technique.

\section{INTRODUCTION}

Totally thoracoscopic mitral valve replacement is one of the most complex operations for totally thoracoscopic cardiac surgery. The heart is located in a special position in the middle mediastinum between the 2 lungs, with complex surrounding structures. Constrained by a series of challenges,

Received May 13, 2021; accepted Fune 10, 2021.

Correspondence: Xiao-fu Dai, Department of Cardiovascular Surgery, Union Hospital, Fujian Medical University, Fuzhou, 350001 People's Republic of China (e-mail: gzlde0323@msn.com). such as $2 \mathrm{D}$ vision, special operation angle, and operational inconvenience in endoscopic surgery, surgeons must be experienced in traditional mitral valve surgery to perform a totally thoracoscopic mitral valve surgery. Skilled surgeons with a good knowledge of anatomy and tacit surgical team cooperation are required.

The first minimally invasive mitral valve surgery (MIMVS) was performed in 1996 [Navia 1996]. To date, totally thoracoscopic cardiac surgery has advanced rapidly, and cardiac surgeons worldwide have reported surgical experiences with encouraging results. Most of these results indicate that MIMVS provides excellent and clear exposure to the mitral valve, with mortality and complication outcomes comparable to those obtained with traditional methods [Svensson 2010; Al Otaibi 2017; Ryan 2005; Greco 2008].

Several previous studies showed that totally thoracoscopic cardiac surgery has advantages over traditional strategies, including less intraoperative blood loss, less pain, better cosmesis, and shorter hospital stay. In recent years, totally thoracoscopic cardiac surgery has gradually become a standard procedure for mitral valve disease [Gammie 2010; Cohn 1997; Walther 1999].

Cumulative sum analysis (CUSUM), a sequential analysis method, was introduced into clinical analysis in the 1970 s [Chaput de Saintonge 1974]. In this paper, CUSUM was used to analyze the learning curve from 100 cases of totally thoracoscopic mitral valve surgeries carried out by a single surgeon.

\section{[HI]METHODS}

\section{General Information}

From May 31, 2019, to June 4, 2020, in the cardiovascular surgery department of Fujian Union Hospital, a total of 100 consecutive totally thoracoscopic mitral valve surgeries were performed by the same surgeon. Inclusion criterion: patients who underwent totally thoracoscopic mitral valve replacement. Exclusion criteria: (1) patients with contraindications for thoracoscopic surgery, (2) patients with severe systemic coexisting diseases, (3) patients with other concurrent cardiac surgery, or (4) patients who were operated by other surgeons. According to these criteria, 100 cases were included in the study.

\section{Surgical Methods}

All patients were in supine position. A double-lumen endotracheal intubation was performed under general anesthesia, and transesophageal echocardiography (TEE) was routinely 
placed. The right hemithorax was slightly elevated $20^{\circ}$ to $30^{\circ}$ with the hips flat and the right elbow bent. Cardiopulmonary bypass (CPB) was established through the femoral artery and vein assisted by a single 2 -stage venous cannula.

Totally thoracoscopic mitral valve replacement was performed using the 3 -ports approach at the right thoracic wall, with the fourth intercostal space under the armpit as the observation portal, the second intercostal space beside the sternum, and the fifth intercostal space in the midline of the clavicle as the operation portal [Bonaros 2006; Ma 2011].

After transthoracic clamp to occlude the ascending aorta and anteriorly, histidine-tryotophan-ketoglutarate perfusion was applied, a single 2-stage cannula was suspended, the right atrium was opened, and the left atrium was entered through an atrial septal incision [Murzi 1997]. Mitral valve plasty or mitral valve replacement was performed after evaluation. After routine TEE and complete evacuation of endocardial gas, the CPB was disconnected and the incision was closed.

\section{Observed Indicators}

(1) General conditions: age, sex, and preoperative cardiac function; (2) indicators related to surgery: operation time, CPB time, cross-clamp time, intraoperative injury, and conversion to opening; and (3) postoperative recovery-related indicators: amount of drainage, oxygenation index (24 hours), serum creatinine (24 hours), and postoperative hospital stay.

\section{CUSUM Analysis}

SPSS17 software was used to arrange all cases in sequence according to the date of surgery. The value of the first case (CUSUM1) is the difference between the operation time $(\mathrm{OT})$ of the first case (OT1) and the average operation time of all cases (OTmean), that is: CUSUM1 $=(\mathrm{OT} 1 \neg-$ OTmean). The value of the second case and each subsequent case (CUSUMn) is the difference between the operation time of that case (OTn) and the average operation time (OTmean), plus the CUSUM value of the previous case, that is: CUSUMn $=($ OTn - OTmean $)+\operatorname{CUSUM}(\mathrm{n}-1)$. According to this rule, CUSUM is accumulated continuously until the CUSUM of the last case is 0 .

\section{Learning Curve Fitting}

The number of cases was taken as the abscissa, and the value of CUSUM was used as the ordinate. Excel 2016 was applied to draw the scatter diagram of the learning curve, and SPSS version 17 software was used to fit the CUSUM learning curve. The fitting model test was judged by $P$ value, and the curve fitting was successful when $P<.05$. The coefficient R2 was used to determine the goodness of fit. The closer $\mathrm{R}^{2}$ is to 1 , the higher the goodness of curve fitting is. The model with the highest $\mathrm{R}^{2}$ is the best fitting model. The CUSUM fitting curve vertex was then taken as the boundary to divide the learning curve into 2 stages. The abscissa value corresponding to the vertex of the curve is the lowest number of surgical cases that a surgeon needs to accumulate to overcome the learning curve.

\section{Statistical Analysis}

SPSS version 17.0 statistical software was used for data analysis. The clinical data of each group were statistically analyzed and compared according to different stages of learning curve. Normal distribution measurement data were expressed as mean \pm standard deviation. Two independent-sample $t$ tests were used to compare the mean between groups. $\chi^{2}$ test was used to compare the count data between groups. $P<.05$ was considered statistically significant.

\section{Results}

SPSS version 17.0 software was used for CUSUM learning curve fitting. $P$ values of the quadratic curve and the cubic curve fitting model were all $<.05$. The goodness-of-fit coefficient $\mathrm{R}^{2}$ values were 0.940 and 0.968 , respectively. The optimal fitting model was judged to be a cubic curve, and the goodness-of-fit coefficient $\mathrm{R}^{2}$ was 0.968 . The fitting equation is CUSUM $(\mathrm{min})=0.008 \times 3-2.292 \mathrm{x}^{2}+145.138 \mathrm{x}+635.666$, where $x$ is the number of surgical cases. The fitting curve crosses the vertex when the number of cases accumulates to the 40th case; taking this as the boundary, the learning curve is divided into stages A and B, respectively. Stage A is the skill acquisition stage, stage $B$ is the proficiency stage, and 40

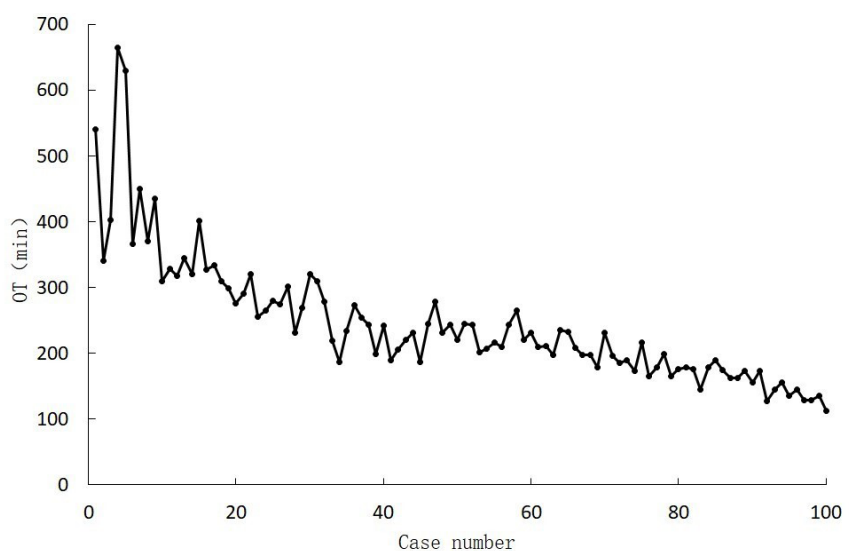

Figure 1. Variation trend of operative time (OT).

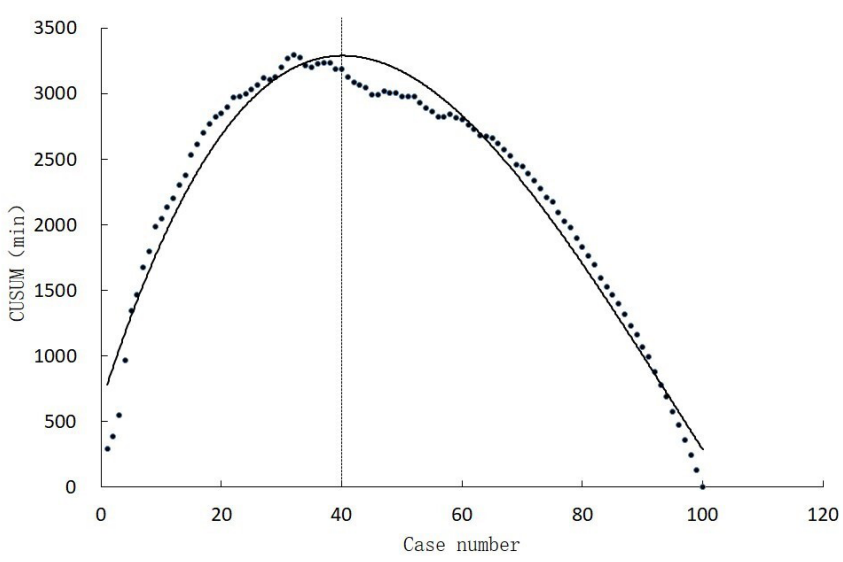

Figure 2. Scatter diagram of CUSUM Learning curve. The solid line is the best fitting model-cubic curve, and the vertical dashed line shows that the corresponding horizontal coordinate of the curve vertex is 40 cases. 
cases is the minimum number needed to cross the learning curve (Figure 2).

A total of 100 cases were included in this study, including 41 males and 59 females. The results in Table 1 showed that the distribution of age and sex in the skill acquisition stage and the proficiency stage was balanced. No differences were observed in the conversion rate to median sternotomy and left ventricular ejection fraction between the 2 stages. But operation time, CPB time, cross-clamp time, volume of drainage, length of hospital stay, oxygenation, serum creatinine levels at 24 hours, and intraoperative injury rates were significantly different between the 2 stages. Operative time, $\mathrm{CPB}$ time, cross-clamp time, volume of drainage, hospitalization time, serum creatinine level (24 hours), and intraoperative injury rates in the skill acquisition stage were all higher than those in the proficiency stage, whereas the oxygenation index in the skill acquisition stage was lower than that in the proficiency stage.

\section{DISCUSSION}

With the continuous improvements in thoracoscopic surgery and the refinement of related instruments, thoracoscopic surgery has been developing rapidly in cardiac surgery. Totally thoracoscopic mitral valve surgery has multiple advantages, including excellent cosmetic outcome, clear intraoperative exposure, enlarged visual field, small amount of bleeding, and short recovery time [Al Otaibi 2017; Walther 1999; Modi 2008; Ritwick 2008], making it more and more common in the field of cardiac surgery.

However, thoracoscopic cardiac surgery is associated with longer CPB and aortic occlusion times [Modi 2008]. This is mainly due to limited working space and the use of new instruments and limitations of camera technology. In addition, the surgeon needs to be familiar with new cannulation techniques and transthoracic aortic occlusion techniques, and the valve of the patient needs to be accessed from different angles. Although totally thoracoscopic mitral valve surgery was often used in dedicated high-turnover centers and proved to be effective and repeatable, the technique remains difficult to master as a new approach for surgeons who are familiar with traditional approaches [Anyanwu 2012].

This study used the concept of a learning curve. The learning curve is often used in the literature to describe the process of mastering new methods. With the learning curve, one can quantitatively understand the whole process of mastering a new technique. More importantly, we could also avoid unnecessary repetition and mistakes during the study of a new surgical procedure based on the information from a learning curve. In an online survey of 20 experienced surgeons, $90 \%$ believed that $>20$ cases were enough, and 2 surgeons believed that 10 cases were enough, to overcome the learning curve subjectively [Misfeld 2013]. However, the learning curve

Table 1. Comparison of clinical data between 2 stages $\left(x^{-} \pm s\right)$

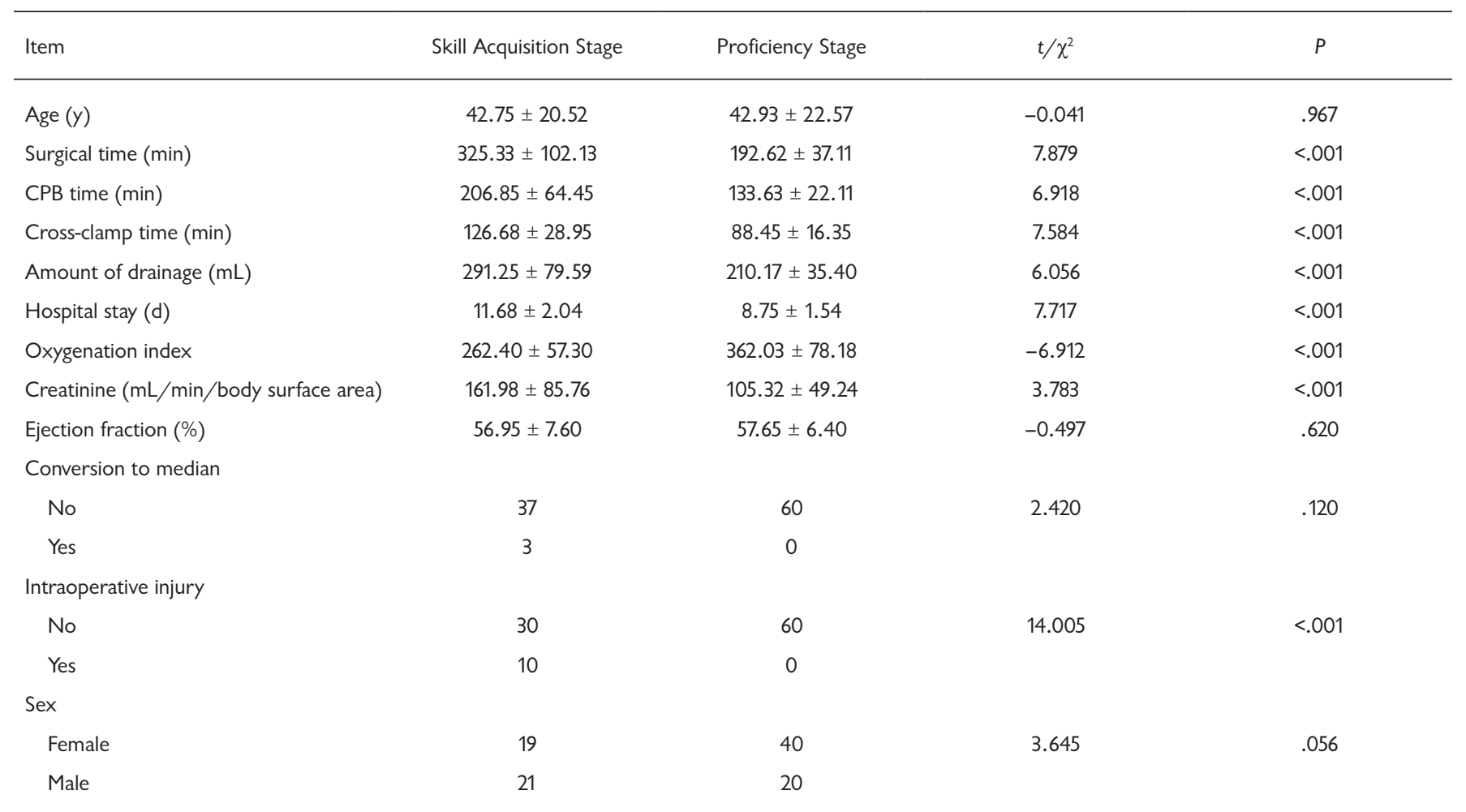

Data are mean \pm standard deviation. 
obtained by the cumulative sum analysis in this study shows that $\geq 40$ cases are needed to overcome the learning curve.

For surgeons preparing for or performing TTMVR surgery, there is an urgent need to understand the learning curve. We applied the CUSUM analysis to evaluate the learning curve of surgery in detail. This method has been used in many studies to calculate the number of cases needed to adopt a new technology [Liu 2018; Holzhey 2013; Wu 2019]. In the CUSUM analysis, the formula: CUSUMn $=(\mathrm{OTn}-$ OTmean $)+\operatorname{CUSUM}(\mathrm{n}-1)$ is used, and the cumulative sum of each operation (CUSUMn) was calculated based on the obtained standardized operation time.

From the scatter diagram of CUSUMn and the number of surgical cases, it can be preliminarily observed that the standardized operation time presents a downward trend with the increase of the number of surgical cases. SPSS software was used to perform curve fitting for the obtained scatter plot, and the best fitting curve was the cubic curve $\left(\mathrm{R}^{2}=0.968, P<.05\right)$. The learning curve of 100 MIMVS peaked when the number of surgical cases accumulated to 40. Taking the 40th case as the cutoff point, the learning curve is clearly divided into 2 stages: the first stage is the skill acquisition stage, and the second stage is the proficiency stage. Before reaching 40 cases is the stage of skill acquisition: during this period, with the accumulation of surgical cases, the operator's surgical skills and experience rapidly improved, and the learning curve showed a steep rise. After 40 cases, the proficiency stage began; that is, the operative technique gradually became mature and relatively stable, the operation time tended to be relatively stable, and the operation outcome was good. Therefore, 40 cases of operation are required for surgeons to overcome the learning stage and enter the proficiency stage in totally thoracoscopic mitral valve replacement surgery.

We have reviewed studies on learning curves, and the number of cases that MIMVS pay to overcome them varies in different studies [Holzhey 2013; Wu 2019; Rogers 2004]. All studies showed that with the improvement of surgeons' case by case and surgical proficiency, the patients' CPB time, operation time, and the incidence of surgical complications were significantly reduced [Holzhey 2013; Nissen 2019].

In this study, we statistically analyzed the operative parameters and perioperative parameters of the skill acquisition stage and the proficiency stage. We found that with the increase in the cumulative number of surgical cases, CPB and cross-clamp times and the incidence of surgical complications were significantly reduced. The comparison data of the 2 stages showed that the general preoperative conditions of the patients were not significantly different. However, with the gradual improvement of the operator's technical level, operation time, CPB time, and cross-clamp time were gradually shortened, and the difference was statistically significant. This is related not only to the master surgeon's technical maturity, but also to the master surgeon's tacit cooperation with the first assistant and even the second assistant (mirror holder). All cases in this study were performed by the same surgeon and first assistant, and the second assistant was rarely changed, which can accelerate the growth of the master surgeon and assistant.
As the operation time was significantly shortened in the proficiency stage, the drainage volume, pulmonary oxygenation and creatinine levels were also significantly improved in the second stage, suggesting that the operation time was related to the perioperative recovery of the patients. The length of hospital stay was shortened from $11.68 \pm 2.04$ to $8.75 \pm 1.54$ days, and the difference between the 2 stages was statistically significant. This is likely related to o the improvement of technical level as well as an increase in the understanding of the management of patients after totally endoscopic mitral valve surgery.

Thus totally thoracoscopic mitral valve replacement is safe and feasible. Due to the complexity and precision of the operation and the high requirements for endoscopic techniques, the learning curve of the operation is necessarily a long process of gradual progress. The CUSUM surgical learning curve of totally thoracoscopic mitral valve surgery can be best described by the cubic curve, which can be clearly divided into an ascending stage and a descending stage, corresponding to the skill acquisition stage and the proficiency stage, respectively. The number of surgeries that a surgeon needs to accumulate from the learning stage and enter the proficiency stage of MIMVS is 40.

The limitations of this study are that it reports only 1 surgeon's experience in a single center, and the number of cases is still limited. In spite of these limitations, our study still has its clinical significance. With the increase of the number of surgical cases, the proficiency of surgeons will be improved continuously.

\section{Conclusions}

Totally thoracoscopic approach is an excellent choice for mitral valve replacement and provides excellent short-term outcomes. For surgeons experienced in traditional mitral valve surgery, the number of cases needed to accumulate TTMVR from the learning stage to the proficiency stage is 40 , according to our research.

\section{ACKNOWLEDGMENTS}

We earnestly acknowledge the contribution by the participating doctors, Xue-shan Huang, Han-fan Qiu, and Dongshan Liao.

\section{REFERENCES}

Al Otaibi A, Gupta S, Belley-Cote EP, et al. Mini-thoracotomy vs. conventional sternotomy mitral valve surgery: a systematic review and metaanalysis. J Cardiovasc Surg (Torino) 2017;58:489-496.

Anyanwu AC, Adams DH. Should complex mitral valve repair be routinely performed using a minimally invasive approach? Curr Opin Cardiol 2012;27:118-124.

Bonaros N, Schachner T, Oehlinger A, et al. Robotically assisted totally endoscopic atrial septal defect repair: insights from operative times, learning curves, and clinical outcome. Ann Thorac Surg 2006;82:687-693.

Chaput de Saintonge DM,Vere DW. Why don't doctors use cusums? 
Lancet 197:1:120-121.

Cohn LH, Adams DH, Couper GS, Bichell DP, Rosborough DM, Sears SP, Aranki SF. Minimally invasive cardiac valve surgery improves patient satisfaction while reducing costs of cardiac valve replacement and repair. Ann Surg 1997;226:421-426.

Gammie JS, Zhao Y, Peterson ED, O’Brien SM, Rankin JS, Griffith BP. J. Maxwell Chamberlain Memorial Paper for adult cardiac surgery. Lessinvasive mitral valve operations: trends and outcomes from the Society of Thoracic Surgeons Adult Cardiac Surgery Database. Ann Thorac Surg 2010;90:1401-1408, 1410.e1.

Greco E, Zaballos JM, Alvarez L, Urso S, Pulitani I, Sàdaba R, Juaristi A, Goiti JJ. Video-assisted mitral surgery through a micro-access: a safe and reliable reality in the current era. J Heart Valve Dis 2008;17:48-53.

Holzhey DM, Seeburger J, Misfeld M, Borger MA, Mohr FW. Learning minimally invasive mitral valve surgery: a cumulative sum sequential probability analysis of 3895 operations from a single high-volume center. Circulation 2013;128:483-491.

Liu X, Chen X, Shen Y, et al. Learning curve for uniportal video-assisted thoracoscopic surgery lobectomy-results from 120 consecutive patients. J Thorac Dis 2018;10:5100-5107.

Ma ZS, Dong MF, Yin QY, Feng ZY, Wang LX. Totally thoracoscopic repair of atrial septal defect without robotic assistance: a single-center experience. J Thorac Cardiovasc Surg 2011;141:1380-1383.

Misfeld M, Borger M, Byrne JG, et al. Cross-sectional survey on minimally invasive mitral valve surgery. Ann Cardiothorac Surg 2013;2:733-738.

Modi P, Hassan A, Chitwood WR Jr. Minimally invasive mitral valve surgery: a systematic review and meta-analysis. Eur J Cardiothorac Surg 2008;34:943-952.
Murzi M, Kallushi E, Solinas M, et al. Minimally invasive mitral valve repair using transthoracic aortic occlusion. Ann Thorac Surg 1997;63:1477-1479.

Navia JL, Cosgrove DM 3rd. Minimally invasive mitral valve operations. Ann Thorac Surg 1996;62:1542-4.

Nissen AP, Nguyen S, Abreu J, Nguyen TC. The first 5 years: building a minimally invasive valve program. J Thorac Cardiovasc Surg 2019;157:1958-1965.

Ritwick B, Chaudhuri K, Crouch G, Edwards JR, Worthington M, Stuklis RG. Minimally invasive mitral valve procedures: the current state. Minim Invasive Surg 2013;2013:679276.

Rogers CA, Reeves BC, Caputo M, Ganesh JS, Bonser RS, Angelini GD. Control chart methods for monitoring cardiac surgical performance and their interpretation. J Thorac Cardiovasc Surg 2004;128:811-819.

Ryan WH, Dewey TM, Mack MJ, Herbert MA, Prince SL. Mitral valve surgery using the classical 'heartport' technique. J Heart Valve Dis 2005;14:709-714.

Svensson LG, Atik FA, Cosgrove DM, et al. Minimally invasive versus conventional mitral valve surgery: a propensity-matched comparison. J Thorac Cardiovasc Surg 2010;139:926-932.e1-e2.

Walther T, Falk V, Metz S, Diegeler A, Battellini R, Autschbach R, Mohr FW. Pain and quality of life after minimally invasive versus conventional cardiac surgery. Ann Thorac Surg 1999;67:1643-1647.

Wu X, Wei W, He Y, Qin H, Qi F. Analysis of the learning curve in mitral valve replacement through the right anterolateral minithoracotomy approach: a surgeon's experience with the first 100 patients. Heart Lung Circ 2019;28:471-476. 